\title{
THROMBOEMBOLIC PROPHYLAXIS FOR ONCOLOGICAL PATIENTS - GENERAL RECOMMENDATIONS
}

\author{
Chernopolsky P., V. Bozhkov, R. Madjov \\ Second Department of surgery, UMHAT "St. Marina" - Varna, Medical university - Varna
}

\section{Reviewed by: Assoc. Prof. R. Radev}

\begin{abstract}
Venous thromboembolism is a common complication of surgery, occurring in up to $65 \%$ of patients undergoing orthopedic surgery and $33 \%$ or more of patients undergoing general surgical procedures. Evidence-based guidelines recommend that acutely ill hospitalized medical patients who are at risk of venous thromboembolism (VTE) should receive prophylaxis. VTE includes two closely related clinical manifestations: deep venous thrombosis (DVT), usually of the lower limbs, and pulmonary embolism (PE). VTE remains a burdensome source of morbidity and mortality despite many advances in diagnostic and treatment tools - in USA - about 1,8 - 2,0 million patients per
\end{abstract}

Table 1: Risk factors for venous thromboembolism (VTE)

\begin{abstract}
year and in Europe - 650-700,000 patients. VTE occurs in $10-80 \%$ of hospitalized patients depending on the reason for hospitalization. Because few specific symptoms or findings may occur due to DVT or PE, clinical diagnosis is unreliable. Routine screening of all patients is not practical. However, effective methods of prophylaxis are both cost-effective and safe. Barriers to widespread application include the perception that the incidence of DVT/PE is low, that bleeding complications are frequent and that cost of prevention is high. For these reasons, systematic pharmacological prophylaxis in patients at high risk for venous
\end{abstract}

\begin{tabular}{|c|c|}
\hline $\operatorname{Age}^{1}$ & $\begin{array}{l}\text { Exponential increase in risk with age. In the general population: } \\
<40 \text { years - annual risk } 1 / 10,000 \\
60-69 \text { years - annual risk } 1 / 1,000 \\
>80 \text { years - annual risk } 1 / 100 \\
\text { May reflect immobility and coagulation activation }\end{array}$ \\
\hline Obesity & $\begin{array}{l}3 \mathrm{x} \text { risk if obese (body mass index }>=30 \mathrm{~kg} / \mathrm{m}^{2} \text { ) } \\
\text { May reflect immobility and coagulation activation }\end{array}$ \\
\hline Varicose veins & $\begin{array}{l}1.5 \mathrm{x} \text { risk after major general / orthopaedic surgery } \\
\text { But low risk after varicose vein surgery }\end{array}$ \\
\hline Previous VTE & Recurrence rate $5 \%$ / year, increased by surgery \\
\hline Thrombophilias & $\begin{array}{l}\text { Low coagulation inhibitors (antithrombin, protein C or S) } \\
\text { Activated protein C resistance (e.g. factor V Leiden) } \\
\text { High coagulation factors (I, II, VIII, IX, XI) } \\
\text { Antiphospholipid syndrome High homocysteine }\end{array}$ \\
\hline Other thrombotic states & $\begin{array}{l}\text { Malignancy } 7 \mathrm{x} \text { risk in the general population } \\
\text { Heart failure } \\
\text { Recent myocardial infarction / stroke } \\
\text { Severe infection } \\
\text { Inflammatory bowel disease, nephrotic syndrome } \\
\text { Polycythaemia, paraproteinaemia } \\
\text { Bechet's disease, paroxysmal nocturnal haemoglobinuria }\end{array}$ \\
\hline Hormone therapy & $\begin{array}{l}\text { Oral combined contraceptives, HRT, raloxifene, tamoxifen } 3 \times \text { risk } \\
\text { High-dose progestogens } 6 \mathrm{x} \text { risk }\end{array}$ \\
\hline Pregnancy, puerperium & $10 \mathrm{x}$ risk \\
\hline Immobility & Bedrest $>3$ days, plaster cast, paralysis), $10 \mathrm{x}$ risk; increases with duration \\
\hline \multicolumn{2}{|l|}{ Prolonged travel } \\
\hline Hospitalisation & Acute trauma, acute illness, surgery $10 \mathrm{x}$ risk \\
\hline Anaesthesia & $2 \times$ general vs spinal / epidural \\
\hline
\end{tabular}


thromboembolism is the most effective approach for reducing morbidity and mortality from this pathology.

Risk factors for the development of VTE include: increasing age, prolonged immobility, stroke or paralysis, previous VTE, cancer and therapeutic interventions, major surgery, trauma, obesity, varicose veins, cardiac dysfunction, central venous catheters, inflammatory bowel disease, nephritic syndrome, pregnancy or hormonal therapy use (Table 1). Patients may have several risk factors and that risk is additive. In addition patients may have predisposing thrombophilic disorders such as activated protein $\mathrm{C}$ resistance, antiphospholipid antibodies, deficiency of proteins (C, S, antithrombin), elevated homocysteine levels. The definition of the risk factors for venous thromboembolism allows adopting the most suitable prophylactic regimen. Determinants for the risk of VTE are patient risk factor, both clinical and molecular, and the clinical setting. Patient clinical risk factors are mainly previous VTE and cancer, followed by the age over 70 , bed rest for longer than 4 days and severe medical illness, such as recent stroke or myocardial infarction. Molecular risk factors can be subdivided into inherited and acquired. Among the inherited ones are antithrombin III, protein $\mathrm{C}$ or $\mathrm{S}$ deficiencies, activated protein $\mathrm{C}$ resistance and hyperhomocysteinemia, while acquired factors include lupus anticoagulant and antiphospholipoid syndrome. The risk connected with the clinical setting mostly surgical setting, is the only risk defined by properly performed epidemiological studies. High-risk clinical settings are major general surgery, orthopedic surgery, elective neurosurgery, spinal cord injury, cancer surgery and multiple traumas. The definition of the risk for preoperative VTE is mainly based on clinical risk factors that are present before surgery.

Categorizing patients into different risk classes would allow adopting different prophylaxis measures in different patients (Table 2):
The diagnosis of VTE has important clinical implications. In a prospective observational study of ambulatory patients with cancer initiating chemotherapy, venous and arterial thromboembolism together accounted for $9 \%$ of deaths. Cancer diagnosed at the same time as, or within 1 year of an episode of VTE, is associated with a three-fold greater mortality at 1 year. The risk of fatal PE in patients with cancer undergoing general surgery is three-fold greater than in patients without cancer undergoing similar surgery. In addition VTE recurs three-fold more frequently in cancer patients than in patients who do not have a cancer, and requires long-term anticoagulation with a two-fold greater risk of bleeding complications than in patients who do not have a cancer. VTE in patients with cancer also consumes health care resources. In a retrospective analysis, the mean length of DVT - attributable hospitalization was 11 days, and the average cost of hospitalization for the index episode was $\$ 20,065$ in 2002. Reducing VTE in patients with cancer could therefore have a significant impact on morbidity, outcomes, use of health care resources and, above all, mortality.

\section{RISK FACTORS FOR CANCER ASSOCIATED VTE}

The risk of thrombosis differs across various cancer subgroups and over the natural history of the disease. The risk of VTE is highest in the initial period after the diagnosis of malignancy. The association of VTE with specific sites of cancer such as pancreas, stomach, brain, ovary, kidney and lung, and with the presence of metastatic disease, has been well documented. Newer studies suggest a strong association with hematologic malignancies, particularly lymphomas. VTE increases significantly when patients with cancer are hospitalized. A comprehensive list of risk factors associated with VTE in patients with cancer is summarized in Table 3. Symptoms suggestive of DVT include unilateral calf, leg or thigh swelling pain, whereas a diagnosis of

Table 2. Risk categories for VTE

\begin{tabular}{|c|c|c|}
\hline Low risk & Moderate risk & High risk \\
\hline $\begin{array}{l}\text { Patients }<40 \text { yrs } \\
\text { Uncomplicated surgery } \\
\text { Minimal immobility }\end{array}$ & $\begin{array}{l}\text { General surgery in patients }>40 \mathrm{yrs} \\
\text { Acute myocardial infarction } \\
\text { Chronic illness } \\
\text { Leg fracture in a patient }<40 \mathrm{yrs}\end{array}$ & $\begin{array}{l}\text { Surgery for malignant diseases } \\
\text { Previous VTE } \\
\text { Major trauma } \\
\text { Orthopedic surgery }\end{array}$ \\
\hline
\end{tabular}

Venous thromboembolism (VTE) is a major complication of cancer, occurring in $4 \%$ to $20 \%$ of patients, and is one of the leading causes of death in patients with cancer. The risk of VTE including deep venous thrombosis (DVT) and pulmonary embolism (PE) is increased several-fold in patients with cancer. In a population based study, cancer was associated with a 4.1-fold greater risk of thrombosis, whereas the use of chemotherapy increased the risk 6.5-fold. The reported rates of VTE in patients with cancer are believed to be underestimated, given that autopsy rates of VTE can be as high as $50 \%$ compared with clinical rates of $4 \%$ to $20 \%$.
DVT is generally based on a lower-extremity Doppler ultrasound. Symptoms suggestive of a PE include shortness of breath, tachypnea, pleuritic chest pain, a pleural rub, hypoxia, hemoptysis, tachycardia, syncope along with accompanying symptoms, and signs of a DVT or right heart failure. A diagnosis of $\mathrm{PE}$ is generally based on a ventilation/perfusion scan or spiral computed tomography scan. Coagulation disorders are a common problem in neoplastic patients and many factors contribute to increase the risk of thromboembolic events. A hypercoagulable state is induced by malignant cells interacting directly with hemostatic sys- 
tem and activating the coagulation cascade. Diagnosis of idiopathic DVT in the absence of other risk factors could indicate the presence of occult malignant disease. Cancer patients show an increased susceptibility, as compared with the general population, to develop thromboembolic diseases, suggesting that disorders of coagulations are very common in this disease, although clinical symptoms occur less frequently. The association between hypercoagulability and malignancy has been recognized for the first time in 1865 by professor Trousseau, who drew attention to the high incidence of DVT of the extremities in patients with gastric carcinoma. It is now recognized that thromboembolism is one of the most common causes of death in cancer patients. Mucinproducing carcinoma of gastrointestinal tract, ovarian, pancreatic, prostate and lung cancer, acute promyelocytic leukemia are among the malignancies more frequently associated with thromboembolic episodes.

Table 3. Risk factors for VTE in patients with malignant disease

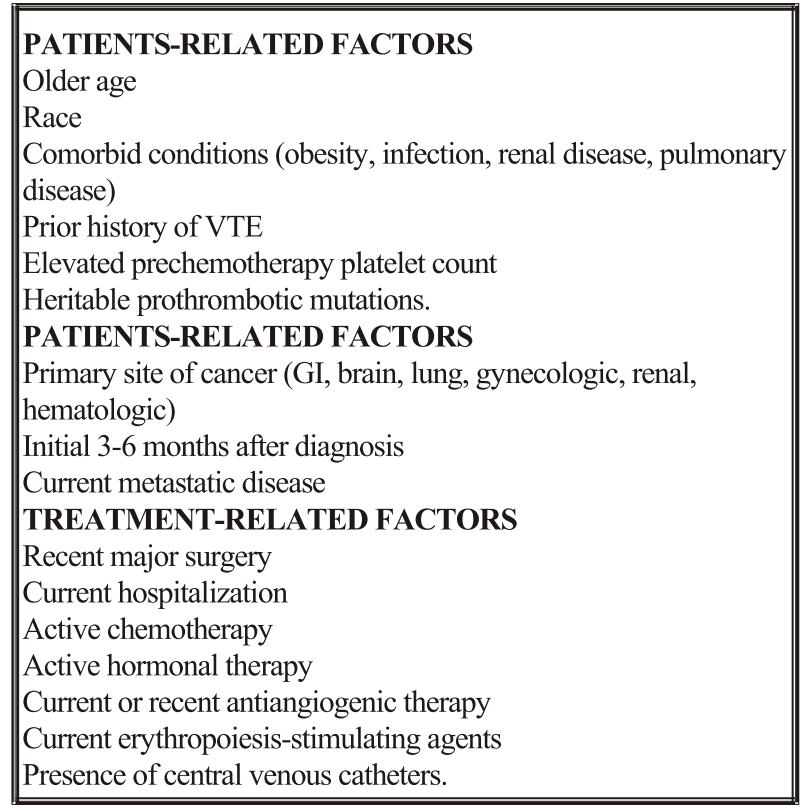

Multiple mechanisms, direct and indirect, are implicated in the pathophysiolofy of coagulation disorders which occur in the neoplastic patient. Malignant cells directly, themselves produce various procoagulant activities (PCAs), among which the best defined are the tissue factor (TF) and the cancer procoagulant $(\mathrm{CP})$. Other PCAs are factor $\mathrm{V}$ receptor associated with tumor cell plasma membrane and factor XIII-like activity capable of fibrin covalent cross linking. In addition tumor cells may secrete a peptide, vascular permeability factor (VPF) or vascular endothelial growth factor (VEGF) which acts as a selective mitogen and chemotactic factor for cultured endothelial cells and induces several genes in these cells, including TF. Platelets also contribute to cancer metastasis enhancing tumor cell interaction with the extra cellular matrix. Among mediators involved in platelet interaction with tumor cells is 12(S)-hydroxyeicosatetraenoic acid, 1(S) -HETE, a metabolite of arachidonic acid. Finally malignant cells contribute to the procoagulant state inducing activation of monocytes, with subsequent expression of TF. Mononuclear phagocytes, like endothelial cells, do not constitutively express TF, but they expose this procoagulant on their surface in response to different stimuli including complement proteins, immune complex and lymphokines. All these effects of malignant cells associated with other factors promoting thrombosis previously mentioned defined the cancer as the best example of "acquired thrombophilia".

\section{Therapeutic agents}

There is general agreement that initiating mechanical preventive methods as soon as risk is identified has tremendous benefit. Elastic stockings and intermittent pneumatic compression are most commonly used and at relatively low cost. The most commonly used methods are low dose unfractionated heparin and low molecular weight heparin. Oral anticoagulants remain in use, especially for orthopedic and stroke patients. Prophylactic methods can be classified into pharmacological agents that interfere with blood coagulation such as heparin and warfarin, or with fibrin stability, such as dextran, and non-pharmacological methods that accelerate venous outflow such as graduated compression elastic stockings or intermittent pneumatic compression. The most effective anticoagulant regimens in the prevention of VTE in high-risk patients are oral anticoagulants, adjusted dose unfractionated heparin (LDUH) and especially low molecular weight heparins (LMWHs). Adjusted dose unfractionated heparin is given usually three times daily by subcutaneous injection at a dose able to maintain the activated partial thromboplastine time (APTT) in the upper limit of the normal range. Adjusted dose oral anticoagulants should be employed in a way as to maintain a targeted international normalized ratio (INR) of 2-3. Adjusted dose unfractionated heparin and oral anticoagulants are effective and relatively safe but require accurate laboratory monitoring to minimize the risk of bleeding side effects. Thus these regimens are rarely adopted and their cost-effectiveness has not even been established. LMWHs are derivatives of unfractionated heparin produced by its depolymerization. LMWHs are as effective and safe as the other two agents but they do not require laboratory monitoring. The introduction into clinical practice of the LMWHs in the past two decades has been one of the most exciting advances in antithrombotic therapy. The main advantages associated with use of LMWHs are that laboratory monitoring is not required and only once daily subcutaneous injections are necessary. Recent studies have suggested that heparin or LMWHs administered to cancer patients may prolong their survival. The administration of heparin should be associated and followed by an oral anticoagulant drug in order to prevent recurrence. As regards the duration of oral anticoagulant therapy, it is a usual practice to continue treatment for three months. Clinical trials have shown that it is advisable in cancer patients to continue anticoagulant therapy beyond three months, because the subject remain at high risk for recurrent thromboembolism, resulting from immobility, procoagulants produced by cancer cells ant the use of chemotherapy. Retro- 
spective studies have reported recurrences rates between $11 \%$ and $42 \%$ in patient with different types of malignancy.

\section{New approaches}

In recent years several agents have been conducted on the potential use of anti-aggregation drugs for the prevention and therapy several tumor types. The development of cyclooxygenase 2 (COX-2) inhibitors, as new nonsteroidal anti-inflammatory drugs, suggests a new prospective for the treatment of human cancer. Increases levels of COX-2 have been found in inflammatory states and in neoplastic cells of human colon, breast, prostate and lung cancer and their associated premalignant lesions. Evidence has been provided that COX-2 and COX-2 derived prostaglandins may play a role in development of cancer, through stimulation of tumor cell growth and neovascularization. For example colecoxib shows a potent antiangiogenic activity, suppressing growth of lung and colon tumors implanted into recipient mice.

Although prophylaxis in patients at risk for VTE can minimize mortality and morbidity it is still underutilized. The definition of the risk of postoperative DVT on an individual basis should improve the efficacy of prophylaxis. Recommended prophylactic agents vary in different clinical settings. Hemostatic abnormalities are found in more than $90 \%$ of patients with malignancies and clinically expressed as venous thromboembolic events in $15 \%$ of these patients. Finally with regards to the management of thromboembolism in cancer, these patients must be treated with anticoagulant therapy. A large number of studies have shown that either low molecular weight heparins or standard unfractionated heparin for the treatment of DVT in hospitalized patients are equally safe and effective. Therefore during prolonged immobilization following surgery, anticoagulant therapy must be administered to patients with malignancies because of their increased risk of VTE compared to patients free of cancer.

\section{REFERENCES}

1. Wells PS, Hirsch J, Anderson DR. Accuracy of clinical assessment of deep venous thrombosis. Lancet 1995;345:1326-30.

2. Blatter W, Partsch H, Hertel T. Leitlinien zur Diagnostikund Therapie der tiefen
Bien-Beckenvenenthrombose. Phlebologie 1998;27:84-88

3. Risk of Venous Thromboembolism in Users of Hormone Replacement Therapy Daly, Edel; Vessey, Martin P.; Hawkins, Michael M.; Carson, Jeffrey L.;

Gough, Parimala; Marsh, Sally

4. Carter C, Gent M, Leclerc JR. The epidemiology of venous thrombosis. In: Coleman RW, Hirsh J, Marder VJ, et al, eds.Hemostasis and thrombosis. Philadelphia: JB Lippincott, 1987; 76:1185-98

5. Bergqvist D, Burmark US: Low-molecular-weight heparinstarted before surgery as prophylaxis against deep-vein thrombosis:2500 versus 5000 anti-Xa units in 2070 patients. Br JSurg 82:496-501, 1995.

6. Bertomeu MC, Gallo S, Lauri D Levine MN, et al: Chemotherapy enhances endothelial reactivity to platelets. Clin Expl Metastasis 8:511-518, 1990.

7. Bick RL. Alterations of hemostasis associated with malignancy: etiology, pathophysiology, diagnosis and management. SeminThromb Hemost 5:1-26, 1978.

8. Bottasso B, Mari D, Coppola R, et al: Hypercoagulability and hyperfibrinolysis in patients with melanoma. Thromb Res 81:345-352, 1996.

9. Brown LF, Detmar M, Claffey KP: Vascular permeability factor/vascular endothelial growht factor: a multifunctional angiogenesis factor. In: Goldberg ID, Rosen EM, eds. Regulation of Angiogenesis. Basel Birgkhauser Verlag, 233-269;

10. Buccheri G, Ferrigno D, Ginardi C, et al: Haemostatic abnormalities in lung cancer: prognostic implications. Eur J Cancer 33:50-55, 1997.

11. Calvo FA, Hidalgo OF, Gonzales F, et al: Urokinase combination chemotherapy in small cell lung cancer. A phase II study. Cancer 70:2624-2630, 1992.

12. Carey MG, Rodgers GM: Disseminated intravascular coagulation: clinical and laboratory aspects. Am J Hematol 59:65-73, 1998.

13. Chahinian AP, Propert KJ, Ware JH, et al: A randomized trial of anticoagulation with warfarin and alternating chemotherapy in extensive small-cell lung cancer by the Cancer and Leukemia Group B. J Clin Oncol 7:993-1002, 1989.

14. Chan A, Woodruff RK: Complication and failure of anticoagulation therapy in the treatment of venous thromboembolism in patients with disseminated malignancy. Aust NZ J Med 22:119-122, 1992. 